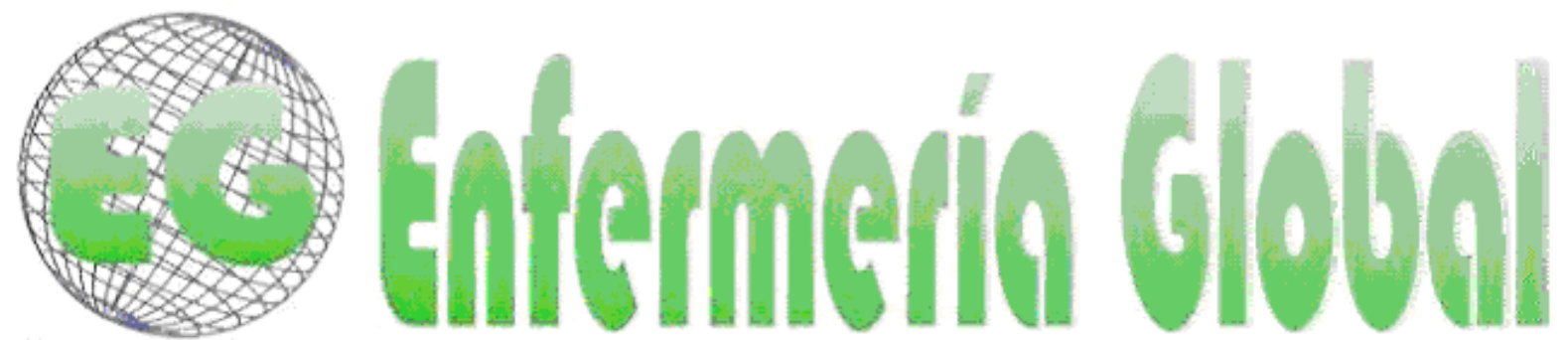

ISSN 1695-6141

Revista electrónica trimestral de Enfermería

$\mathrm{N}^{0} 30$

www.um.es/egloball

Abril 2013

DOCENCIA - INVESTIGACIÓN

\title{
Las relaciones anatómicas involucradas en la administración de medicamentos por vía intramuscular: un campo de estudio de la enfermera
}

As relações anatômicas envolvidas na administração de medicamentos por via intramuscular: um campo de estudo do enfermeiro

The anatomic relationship involved in the administration of medication via the intramuscular route: a field of study of nurses

\section{*Da Silva, Paulo Sérgio **Vaz Vidal, Selma}

*Mestrando del Programa de Post-Graduación de la Universidad Federal del Estado de Rio de Janeíro - UNIRIO. Docente del Centro Universitario Serra dos Órgãos - UNIFESO - Teresópolis - . E-mail: pssilva2008@gmail.com **Máster en Enfermería por la Universidad Federal del Estado de Rio de Janeiro - UNIRIO. Doctoranda del Programa de Post-Graduación en Bioética - PPGBIOS. Docente del Centro Universitario Serra dos Órgãos - UNIFESO - Teresópolis - RJ. Brasil.

\section{Palabras clave:Inyecciones Intramusculares; Relaciones Enfermero - Paciente; Anatomía Regional Palavras chave: Injeções Intramusculares; Relações Enfermeiro-paciente; Anatomia Regional. Keywords: Intramuscular Injections; Nurse - Patient Relationships; Anatomy Regional.}

\section{RESUMEN}

Objetivos: Seleccionar los estudios que relacionan las descripciones anatómicas de los principales músculos que se utilizan en la práctica clínica para la elección de la región de la administración de fármacos y estudios que establecen las relaciones anatómicas entre las estructuras vasculares, nerviosas y músculo esquelético en el proceso de administración de medicamentos por vía intramuscular.

Metodología: Se trata de una revisión sistemática guiada por ocho pasos construidos por el autor que indicaron las fases cubiertas en la selección de los manuscritos para hacer frente a las preguntas de investigación.

Resultados: Dos estudios traían informaciones de la región deltoides sobre la lesión del nervio axilar y su relación con la arteria circunfleja posterior del húmero. En cuanto a la región ventro-glútea se abordaron los dos modelos de localización anatómica más adecuada para la punción de tal manera que no lesionara el nervio glúteo superior y la arteria glútea superior. En relación la región dorsoglútea, los debates se limitan a la conformación entre el nervio ciático y el músculo glúteo mayor, que se 
distribuye ampliamente en la literatura clásica. La parte lateral de la coxa lleva a una reflexión sobre la relación con el nervio cutáneo lateral del músculo.

Conclusión: El estudio presenta de manera sistemática las principales relaciones anatómicas que interferen en el desarrollo de la técnica de la administración de medicamentos por vía intramuscular realizada por la enfermera, que debe adaptarse a las diferentes áreas del conocimiento a la individualidad del cliente.

\section{RESUMO}

Objetivos: Selecionar os estudos que apontam as descrições anatômicas dos principais músculos utilizados na prática clínica para eleição da região de administração de medicamentos e analisar os principais estudos que estabelecem as relações anatômicas existentes entre as estruturas vásculonervosas e a musculatura estriada esquelética, no processo de administração de medicamentos por via IM.

Método: Trata-se de uma revisão sistemática norteada por oito passos construídos pelo autor que indicaram as fases percorridas de seleção dos manuscritos para atender as perguntas do estudo.

Resultados: Na região deltóidea dois estudos trousse informações sobre a lesão do nervo axilar e sua relação com artéria circunflexa posterior do úmero. Quanto à região ventro-glútea foram abordados os dois modelos de delimitação do sítio anatômico mais adequado para punção de tal forma que não lesione o nervo glúteo superior e a artéria glútea superior. No que diz respeito à região dorsoglútea, as discussões ficaram limitadas à conformação existente entre o nervo ciático e o músculo glúteo máximo, o que amplamente esta difundida nas diversas literaturas clássicas. A região lateral da coxa nos remete a uma reflexão acerca da relação com o nervo cutâneo lateral da coxa.

Conclusão: A revisão apresenta de forma sistematizada as principais relações anatômicas que permeiam o desenvolvimento da técnica de administração de medicamentos por via IM realizada pelo enfermeiro, que deve adequar as diversas áreas do conhecimento à individualidade do cliente

\section{ABSTRACT}

Aim: to select the studies that link the anatomical descriptions of the major muscles used in clinical practice for the election of the region of drug delivery and to review key studies that establish the anatomical relationships between the vascular-nervous structures and skeletal striated muscle in the process of administering intramuscular medication.

Methodology: this is a systematic review guided by eight steps constructed by the author that indicated the covered phases in the selection of manuscripts to address the research questions.

Results: Two studies gave information of the deltoid region about the injury to the axillary nerve and its relationship to the posterior humeral circumflex artery. As for the ventral-gluteal region, the two most suitable models of anatomical site most suitable for puncture in such a way that does not injure the superior gluteal nerve and superior gluteal artery were studied. With regard to the region back-gluteal, discussions were limited to the conformation between the sciatic nerve and the major gluteus muscle, which is widely distributed in the various classical literatures. The lateral thigh leads us to a reflection on the relationship with the lateral cutaneous nerve of the thigh.

Conclusion: the review presents in a systematic way the main anatomical relationships that underlie the development of the technique of administering intramuscular medication performed by nurses, which should suit the different areas of knowledge to the individuality of the client.

\section{INTRODUCCIÓN}

El conocimiento anatómico está inserto en un área de estudios denominada morfofuncional, que proporciona ayuda teórica de las diversas estructuras del organismo, así como de su funcionamiento a los profesionales del área de salud. 
La enfermera como integrante del equipo de salud busca articular en su actuación práctica, los princípios morfológicos, humanísticos y técnicos-cientificos para la realización de procedimientos que son de responsabilidad legal.

Entre las varias prácticas que son realizadas en los ambientes terapéuticos bajo la responsabilidad de la enfermera, la administración de medicinas parenterales, sobre todo por vía intramuscular (IM), exige que el profesional tenga una visión científica y analice las regiones anatómicas posibles y adecuadas al cliente, considerando las características farmacológicas de las drogas, la capacidad de absorción muscular y el camino recorrido por el medicamento en su organismo hasta su eliminación.

Las principales regiones de eliminación medicamentosa por vía muscular en el cliente y de mayor implicación clínica en el sentido cráneo-podálico son: deltoides; dorso glúteo; vientre-glúteo y haz lateral del muslo.

El enfermero, al realizar una administración inyectable en el vientre muscular, debe estar atento a los factores generales de variaciones anatómicas como: biotipo, edad, sexo, raza y condiciones locales, que obedecen a características de variaciones individuales de las regiones de implicación clínico-terapéutica, tales como: forma, largura, anchura, espesura y disposición de las fibras musculares.

Los factores mencionados posibilitan la fuga de las características anatómicas esperadas, sin traer cualquier perjuicio funcional al cliente, obligando al profesional a adecuarse a la región más apropiada, a los materiales disponibles y a las características del fármaco prescrito por el médico ${ }^{(1,2)}$.

La administración de medicinas por vía IM es un compromiso del enfermero y de su equipo, luego el proceso medicación-responsabilidad abarca cuestiones de respeto con aquel que recibe la droga, por medio de la interacción cliente-enfermera, lo que resulta en una adecuación de los cuidados a la singularidad de cada cliente.

El cliente es la fuente de cuidado y de los estudios morfológicos, y no está disociado de las cuestiones humanísticas, siendo así, el enfermero al administrar medicinas en la masa muscular, debe asociar las íntimas relaciones anatómicas de estructuras vasculares y nerviosas para alcanzar su bienestar y contribuir al proceso saludenfermedad.

A partir de estas reflexiones, esta investigación presenta como objeto de estudio las relaciones anatómicas comprendidas en la administración de las medicinas por vía IM.

Para lograr tal fin fueron desarrolladas las siguientes cuestiones de esta revisión sistemática: ¿Qué estudios asocian las relaciones anatómicas existentes entre los nervios motores y la musculatura estriada esquelética cuando se administra una medicina en las diversas regiones de la vía IM? ¿Qué trabajos científicos demostraron relaciones de los conocimientos de vascularización en la administración de medicinas en las regiones de la vía IM?

Entender las peculiaridades anatómicas en la realización de una punción muscular lleva a la enfermera a mantenerse siempre actualizada sobre las técnicas que le son de competencia legal, a fin de prestar una asistencia de calidad al cliente que está bajo su responsabilidad. 
Con base en este pensamiento, fueron definidos los siguientes objetivos de este estudio: seleccionar los estudios que señalan las descripciones anatómicas de los principales músculos utilizados en la práctica clínica para elegir la región de la administración de medicinas y analizar los principales estudios que establecen las relaciones anatómicas existentes entre estructuras vásculo-nerviosas y la musculatura estriada esquelética, en el proceso de administración de medicinas por vía IM.

\section{MÉTODO}

El método que nos orientó en esta investigación fue la revisión sistemática, considerada como una actividad fundamental para la práctica basada en evidencia, ya que condensa una gran cantidad de informaciones en un único estudio, tornando de fácil acceso la información, refinando los estudios y separando los de menor rigor académico de los fuertemente confiables, además de servir de base científica para formulación de guías de conductas ${ }^{(3)}$.

Para identificar los trabajos científicos publicados sobre las relaciones anatómicas abarcadas en la administración de medicinas por vía IM, fue realizada revisión sistemática siguiendo los pasos identificados en el cuadro I. 
Cuadro I: Los ocho pasos de la revisión sistemática que establecen las relaciones anatómicas abarcan la administración de medicinas por vía IM.

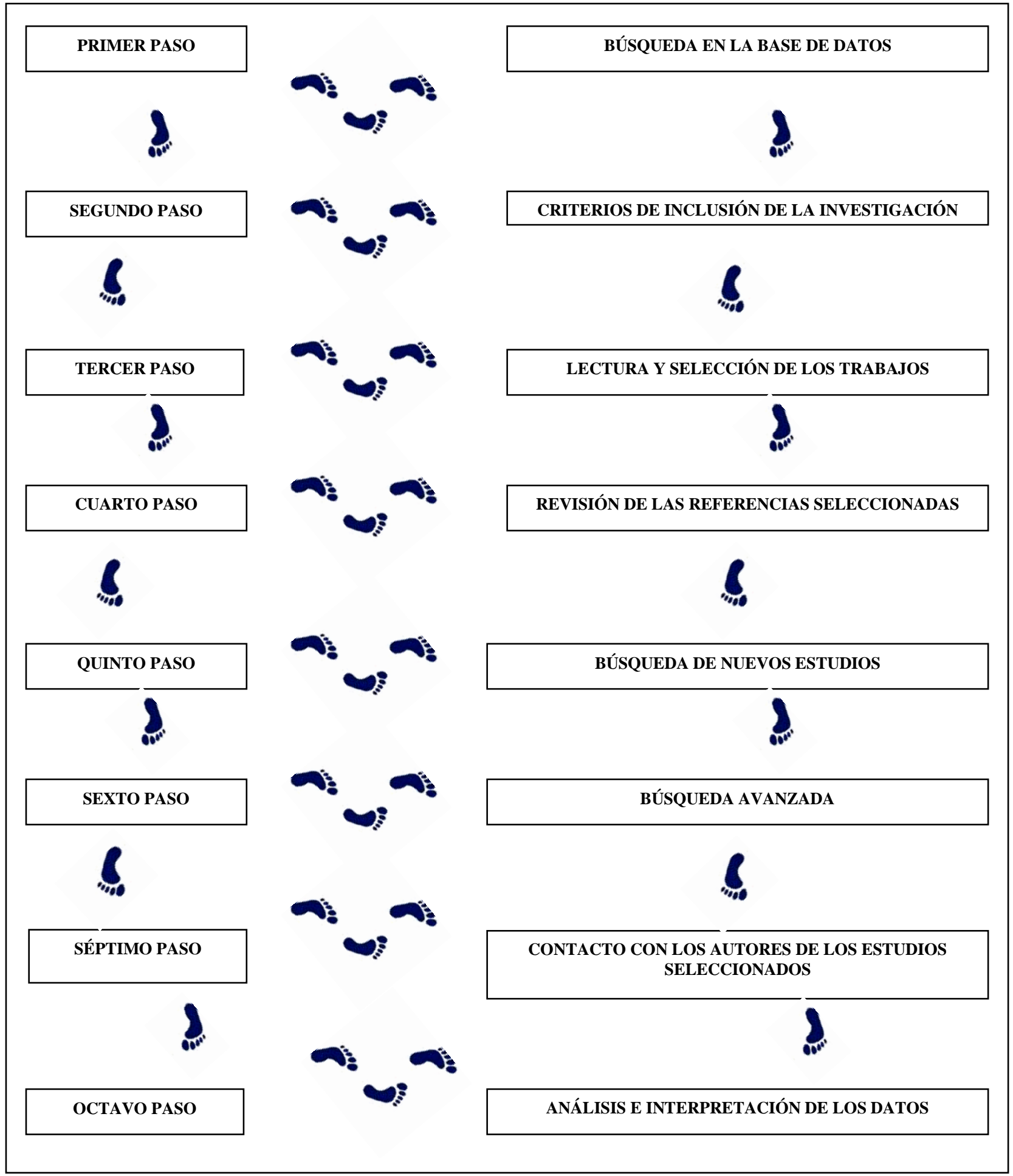

Fuente: Esquematización del autor

La búsqueda fue realizada en la Biblioteca Virtual en Salud con enfoque para base de datos electrónicos de enfermería (BDENF) y Scientific Electronic Library Online (SCIELO). 
La elección de bases por datos BDENF recae en la caracterización de que esa búsqueda es específica de enfermería, mientras que la base de datos SCIELO fue definida por presentar mayor acervo literario sobre la temática abordada. Las referencias de los trabajos científicos que satisfacen los criterios de inclusión fueron evaluadas, independientemente del periódico.

Para la producción de datos de este estudio, se siguieron tres criterios de inclusión: (a) tratar la temática bajo el punto de vista anatómico y temático de esta investigación; (b) publicaciones en el periodo del año 2000 hasta el 2010, periodo de mayor concentración de esos trabajos científicos; (c) investigaciones producidas en Brasil.

Durante las búsquedas, el descriptor en idioma portugués utilizado para la elección de los estudios de interés fue: "inyecciones intramusculares".

Con el fin de auxiliar en la búsqueda de trabajos científicos recurrimos a los operadores lógicos "AND" en un primer momento y al "OR" en un segundo periodo, a fin de conciliar los términos utilizados para la sistematización de las publicaciones.

Se definieron todos los resúmenes de artículos conteniendo los descriptores identificados. Sólo fueron seleccionados los que atendieron la temática en cuestión: "administración de medicinas por vía intramuscular".

Los estudios seleccionados de acuerdo con la temática tuvieron sus referencias revisadas y analizadas para evaluar la presencia de algún estudio que fue publicado en el periodo de tiempo seleccionado, y que por azar no fue aglutinado inicialmente en la revisión. Eso justifica la utilización de tesis para análisis, pues estaban listados en las referencias revisadas y atendieron los criterios de inclusión.

Esa estrategia es considerada otra forma de busca y consiste en el chequeo de las referencias bibliográficas de los estudios identificados, con el propósito de encontrar otros trabajos que tengan los criterios determinados anteriormente ${ }^{(4)}$.

Terminada la fase de análisis de las referencias, se realizó la búsqueda de las referencias seleccionadas, que no figuraban en los resultados inicialmente obtenidos. Ese levantamiento contó con el auxilio de las mismas bases de datos inicialmente consultadas, y las respectivas revistas de publicación de los trabajos científicos.

Con la intención de evitar que estudios potencialmente válidos, que aborden la temática no queden fuera de la revisión, se realizó una nueva consulta en las referidas bases en un segundo momento, pero con la utilización de descriptores más amplios tales como: "enfermero/medicinas", "técnicas/trabajadores/enfermería" y "técnicas/medicinas/errores".

Después de esta búsqueda avanzada todos los artículos no disponibles en las bases virtuales en sites de las respectivas revistas, fueron incluidos en la revisión por medio de consulta en los periódicos de una biblioteca de una universidad situada en el municipio de la región serrana de Rio de Janeiro, que utiliza un sistema de conmutación de artículos entre diversas bibliotecas COMUT.

Los trabajos científicos que disponían de alguna dirección electrónica permitieron el intercambio directo de informaciones entre el autor y los investigadores. De esa manera se objetivó la identificación de estudios válidos para la revisión que aún no 
fueron publicados, y que contemplaron la temática y los criterios de inclusión aquí delineados. No se encontró ningún trabajo en vía de publicación, sin embargo el intercambio de informaciones con los diversos investigadores ayudó en el desarrollo de la temática.

El retorno en este tipo de búsqueda depende de la actitud de los investigadores, los cuales podrán contribuir positivamente compartiendo informaciones valiosas para la revisación sistemática, o no ${ }^{(4)}$.

De esta manera, la descripción de esos ocho pasos objetivó contemplar las dos cuestiones científicas estructuradas motivadoras de este estudio, hecho considerado indispensable para una revisación sistemática de calidad ${ }^{(5)}$.

Así, el análisis contó con un total de 05 estudios que proveen junto con la literatura clásica subsidio teórico morfofuncional y de fundamentos básicos de enfermería para fomentar discusiones relacionadas con la administración de medicinas en la región IM.

\section{RESULTADOS Y DISCUSIÓN DE DATOS}

A partir de las búsquedas con bases en datos científicos, inicialmente 47 publicaciones representaron el universo investigado con fuerte potencial de inclusión para esta revisión, pues abordaban la temática "el enfermero frente a la administración de medicinas".

Después de la lectura de los resúmenes y análisis de los trabajos científicos, ese número fue reducido a 11 , teniendo en cuenta que la vía de administración medicamentosa elegida en este estudio es la IM.

Una vez aplicados los criterios de inclusión, ese número fue redimensionado a 06 componiendo la muestra total a ser discutida en este trabajo. De estos destacan 02 disertaciones, 03 trabajos científicos y 01 relato de caso. En la tabla 1, se presentan informaciones generales de los estudios incluidos en esta revisión. 
Tabla I: Estudios que abordan las relaciones anatómicas en la administración de medicinas por vía IM, de acuerdo con los ocho pasos de la revisión sistemática.

\begin{tabular}{l|l|l|l|l}
\hline PRIMER AUTOR & $\begin{array}{l}\text { REGIÓN } \\
\text { ANATÓMICA }\end{array}$ & $\begin{array}{l}\text { AÑO } \\
\text { PUBLICACIÓN }\end{array}$ & LOCAL & $\begin{array}{l}\text { TIPO } \\
\text { TRABAJO DE }\end{array}$ \\
\hline MENESES A. S. & Ventro - Glútea & 2007 & Brasília & $\begin{array}{l}\text { Rev. Brasileira } \\
\text { de } \\
\text { Enfermagem }\end{array}$ \\
\hline MEIRELLES H. & Deltoideana & 2004 & $\begin{array}{l}\text { Rio } \\
\text { Janeiro }\end{array}$ & $\begin{array}{l}\text { Rev. Brasileira } \\
\text { de Ortopedia }\end{array}$ \\
\hline DUQUE L. V.P. & Deltoideana & 2009 & $\begin{array}{l}\text { Rio de } \\
\text { Janeiro }\end{array}$ & J Vasc Bras \\
\hline KALIL J. M. & $\begin{array}{l}\text { Cuatro } \\
\text { regiones }\end{array}$ & 2003 & Maringá & $\begin{array}{l}\text { Monografia } \\
\text { Especialização } \\
\text { Maringá }\end{array}$ \\
\hline GODOY, S. & Ventro - Glútea & 2002 & $\begin{array}{l}\text { Ribeirão } \\
\text { Preto (SP) }\end{array}$ & $\begin{array}{l}\text { Dissertação } \\
\text { Mestrado USP }\end{array}$ \\
\hline ROCHA R. P. & $\begin{array}{l}\text { Vasto Lateral } \\
\text { del muslo }\end{array}$ & 2002 & $\begin{array}{l}\text { Rev. } \\
\text { Associação } \\
\text { Sédica } \\
\text { Brasileira }\end{array}$ \\
\hline
\end{tabular}

Fuente: Esquematización del autor

En cuanto a las elecciones hechas por los profesionales de las regiones clínicas de administración de medicamentos de la muestra de 06 investigaciones, se encontraron $02(33,3 \%)$ trabajos que abordaron el músculo deltoide, $02(33,3 \%)$ documentos la región ventro-glútea, $01(16,7 \%)$ artículos la región lateral del muslo y $01(16,7)$ estudio abordó de forma general las cuatro regiones.

La discusión y tratamiento de los datos serán de acuerdo con las regiones anatómicas, obedeciendo el sentido cráneo- podálico, en ese sentido fueron creadas cuatro categorías analíticas:

Categoría I: Administración de medicinas en la región de los deltoides y los componentes morfológicos

El músculo deltoides, el primero seleccionado para la discusión de datos, es uno de los sitios anatómicos comprendidos en el proceso de administración de medicinas por vía IM, con implicaciones clínicas en el cuidado realizado por el enfermero y su equipo.

Cabe destacar que la administración en esa estructura es evitada como primera elección, por los profesionales. Eso ocurre debido al mayor porcentaje de complicaciones derivadas de punciones musculares realizadas en ese lugar, cuando comparada a las demás regiones; y a las grandes desventajas anatómicas ${ }^{(6,7)}$.

Entre las desventajas que esa vía puede ofrecer al cliente destacan: pequeño reservatorio de tejido para absorber la medicina lo que imposibilita la introducción de grandes volúmenes en el vientre muscular, intolerancia a sustancias irritables, y la 
posibilidad de lesiones de ramos vasculares y nerviosos que pueden comprometer seriamente la irrigación y el drenaje de estos y la función motora del brazo ${ }^{(2)}$.

La comprensión de las íntimas relaciones anatómicas involucradas en ese proceso se hace necesaria para garantizar la calidad en la administración de la medicina y confianza del cliente que está bajo los cuidados de la enfermería.

De los principales puntos anatómicos abarcados en ese procedimiento, se destacan las relaciones del músculo deltoides con las demás estructuras musculares de la cintura escapular, las estructuras nerviosas provenientes del plexo braquial y los vasos arteriales que juntos son responsables de los movimientos del miembro torácico de la persona.

Entre las varias estructuras musculares que directa e indirectamente influencian el músculo deltoides se destacan: la musculatura toroacoapendicular - "responsable en realizar la conexión del miembro superior con el esqueleto axial anteriormente", espinoapendiculares - "responsable en fijar el miembro torácico al esqueleto axial posteriormente" y por fin, los músculos que dan conformidad al hombro propiamente dicho, y el tercio superior del hombro ${ }^{(1)}$.

En lo que concierne a la intervención, la estructura anatómica responsable en enervar el músculo deltoides, consiste en el nervio axilar, que suple el músculo deltoides, corre transversalmente bajo la cobertura del músculo y se curva alrededor del cuello del húmero. La conciencia de esta localización evita lesiones a este nervio durante la aplicación de inyecciones ${ }^{(8)}$.

Los estudios anatómicos recientes demuestran que esa distancia puede variar de 3 a $7 \mathrm{~cm}$ del acrómio tornando peligrosa la técnica clásica para la integralidad del nervio axilar. Se cree que la inyección intramuscular en el músculo deltoides no debe de ser el sitio de primera elección. El riesgo de complicaciones por la utilización de esa vía es mucho mayor si compara con las otras ${ }^{(7)}$.

Respecto a la vascularización se presume que la sustancia inyectada en el músculo deltoides penetre fortuitamente en las arterias circunflejas del cuello del húmero, alcance la arteria axilar y de ahí camine para uno de sus ramos, eventualmente condicionado por normales variaciones anatómicas de la red arterial axilar $^{(9)}$.

Comprender esas íntimas relaciones morfofuncionales del músculo deltoides permite al enfermero seleccionar el lugar más preciso para una punción muscular, considerando los criterios de variaciones anatómicas del cliente.

La comprensión del enfermero sobre las situaciones anátomico-funcionales de la región de los deltoides para la administración de medicinas converge para la interación con el cliente, en las consideraciones de las variaciones individuales que tornan indispensables el toque, teniendo en vista la evaluación de las circunstancias que se presentan.

Categoría II: La región ventro-glútea y sus propuestas de delimitación para la administración de medicinas

Esa categoría nos conduce a la reflexión de la administración en la región ventroglútea, más específicamente en los músculos glúteos medios y mínimos. 
En cuanto a su localización el musculo glúteo mínimo y la mayor parte del músculo glúteo medio, se sitúan profundos al muslo glúteo máximo en la cara externa del íleo. Ellos ejercen un papel esencial durante la locomoción y son ampliamente responsables de impedir la caída de la pelvis en el lado no sustentado al caminar ${ }^{(8)}$.

De las características anatómicas contextualizadas a partir de una base histórica que considera la delimitación de la región ventro-glútea con la mano contra-lateral del examinador evidencia: profundidad terminada en hueso, ausencia de vasos y nervios significantes, imposibilidad de que vasos o nervios sean afectados, incluso en intentos en los que se maneje mal la aguja ${ }^{(10)}$.

A la luz de la anatomía descriptiva y topográfica a una propuesta de un modelo de delimitación geométrica para la administración de medicinas inyectables, en la región ventro-glútea. A partir de esa propuesta, hay una discusión de que el sitio de la punción no varió tomando como referencia la utilización del triángulo que presenta como vértices los siguientes puntos anatómicos: cresta ilíaca antero-posterior, margen posterior del tubérculo ilíaco y; trocánter mayor del fémur. Mientras que al utilizar la mano no hubo relación de proporción entre el tamaño del cuadril y el biotipo de las piezas $^{(11)}$.

Así inferimos que los modelos de eliminación de la región vientre-glútea se complementan correspondiendo al profesional basado en sus conocimientos anatómicos-funcionales delimitar el sitio de la punción más apropiado a ser realizado en el cliente, a fin de que no ocurran lesiones vásculo-nerviosas.

En cuanto a la intervención los músculos glúteos medio y mínimo son enervados por el nervio glúteo superior que tiene su origen en el plexo lumbosacral ${ }^{(1)}$.

En los aspectos de posibilidad de error de punción muscular, en la comparación entre el modelo tradicional de posicionamiento de la mano (de Hochestter) y la del modelo geométrico se agrega la variable biotipo de la persona como un factor determinante para no ocurrir accidentes ${ }^{(11)}$.

Una de las causas posibles de accidentes en esa región anatómica consiste en la sección total o parcial del nervio glúteo superior y ocurre una pérdida motora característica, resultando principalmente en abducción debilitadora del músculo glúteo mediano $^{(8)}$.

Otra posibilidad de accidente en ese sitio anatómico consiste en lesionar estructuras vasculares tales como la arteria glútea superior que es responsable de la irrigación de ese segmento que anatómicamente corre lateralmente, entre los músculos glúteos medianos y mínimos, junto con el ramo profundo del nervio glúteo superior ${ }^{(1)}$.

Tanto el modelo de delimitación como el geométrico de la región ventro- glútea para punción intramuscular, ofrecen menor riesgo para accidentes. Sin embargo, esa región continúa siendo la menos utilizada ${ }^{(11,6)}$.

El hecho es que el enfermero al realizar la técnica en esa región se encuentra frente a un cliente ansioso frente al procedimiento, por lo que se torna imprescindible explicar la importancia del mismo y la palpación de preeminencias óseas para la localización del lugar de la punción. 
Categoría III: Región dorso glútea: un campo elucidado en las literaturas clásicas.

La revisión aquí conducida señaló pocos trabajos científicos que abordasen esa región desde el punto de vista anatómico.

Una de las características morfofuncionales encontradas consiste en la lesión del nervio ciático, que está bastante aclarada en las literaturas clásicas de enfermería.

Esta complicación puede ser evitada con punciones musculares en el cuadrante superior lateral del glúteo en la región dorso glútea que está compuesta por el glúteo máximo ${ }^{(12)}$.

Tal afirmación sale al encuentro de las literaturas actuales de semiotecnia en enfermería, que señalan que el cuadrante superior externo es el mejor sitio de punción, eso ocurre debido al lugar que permite la prevención de lesiones de vasos y nervios $^{(2,13,14)}$.

Esa región es la mejor elección debido a que la inyección en el cuadrante superiormedio proporciona posibilidades de alcanzar los vasos y el nervio glúteo o la división fibular, eventualmente alta, del nervio isquiático ${ }^{(1)}$.

En cuanto a las características musculares del glúteo máximo presenta un vientre muscular bien distribuido, lo que garantiza un reservatorio de tejidos considerable para la absorción medicamentosa, eso se debe a la grande y vasta circulación colateral existente alrededor de la articulación de la cadera, denominada de anastomosis cruciformes ${ }^{(1)}$.

Partiendo de esas acepciones se percibe que la región está bien difundida y conocida por los profesionales de la salud, sin embargo cabe resaltar las peculiaridades morfo funcionales que deben ser consideradas para evitar accidentes al cliente bajo cuidados de enfermería.

Categoría IV: Región vasto lateral del muslo y los cuidados con la intervención cutánea

La última región a ser considerada en esta revisación consiste en el lado lateral del muslo, señalado como un local de elección medicamentosa desde 1920, frente a las contraindicaciones de las regiones dorso glútea y deltoides ${ }^{(2)}$.

El músculo vasto lateral del muslo es uno de los responsables en componer el músculo cuádriceps del muslo, se presenta lateralmente dispuesto al músculo recto del muslo, que está situado anteriormente a esa región ${ }^{(8)}$.

Esa característica anatómica es importante una vez que al administrar una medicina en la porción mediana del músculo vasto lateral del muslo, se hace necesario que el profesional de salud localice el surco muscular responsable en separar esas estructuras íntimas, a fin de que no ocurra cambio de sitio anatómico durante el desarrollo de la técnica.

Se cree que la porción superior del tercio mediano del muslo, en el área preconizada por la técnica clásica (rectángulo $15 \mathrm{~cm}$ abajo del trocánter mayor del fémur, $12 \mathrm{~cm}$ arriba de la rodilla y 7 a $10 \mathrm{~cm}$ de anchura), sería la región menos indicada para 
aplicación de inyecciones intramusculares, pues esta presenta intensa trama nerviosa. Entretanto, la porción inferior de esta misma área, por no presentar ramos nerviosos significantes, puede estar indicada como región de elección para aplicación IM en la región lateral del muslo, lo que posibilita una disminución de los factores álgicos ${ }^{(15)}$.

Partimos del presupuesto de que la porción distal del musculo vasto lateral del muslo tiene una disminución de los ramos nerviosos cutáneos provenientes del nervio cutáneo lateral del muslo, favoreciendo así mayor efectividad técnica por el profesional que la desarrolla.

En cuanto a la vascularización y la intervención motora, ambas estructuras están dispuestas profundamente en el sentido medial, lo que dificulta realmente accidentes de punción muscular.

Por ser una región donde el cliente estará inspeccionando toda la técnica, se hace necesario que el profesional analice el mejor lugar terapéuticamente, a fin de disminuir las angustias y aflicción frente el procedimiento.

\section{CONCLUSIÓN}

Los estudios que abordan administración de medicinas por vía IM, inclusos en esa revisión sistemática apuntan la escasez de referencias de la temática bajo el punto de vista anatómico.

Se percibe que los estudios aquí seleccionados son abordados por las diversas áreas profesionales, tales como enfermeros, médicos, fisioterapeutas y farmacéuticos. Este hecho nos lleva a una reflexión sobre el proceso multidisciplinar del trabajo de salud. Es relevante la producción de la investigación en la enfermería, pues es esta la que ayudará la práctica del enfermero.

La revisión demostró que la investigación sobre la administración de medicinas en el músculo deltoides se presenta íntimamente relacionada con el nervio axilar y con las arterias circunflejas (anterior y posterior del húmero). Siendo así, esa región se torna peligrosa ya que puede producir lesiones motoras y tisulares considerables, debido a la superficialidad e imprecisión anatómica de tales estructuras, según los autores investigados.

En cuanto a la región ventro-glútea aunque sea la menos utilizada por los profesionales, es la que más presentó estudios que articulan una reflexión a la luz de la anatomía. La técnica de delimitación del sitio de la punción muscular fue puesta a partir del modelo tradicional, o sea, con el auxilio de las manos, y a partir de la propuesta del modelo de delimitación geométrica. Los estudios demuestran que el modelo geométrico permite mayor exactitud en el lugar de la punción, evitando accidentes en estructuras nerviosas (nervio glúteo superior) y vasculares (arteria glútea superior).

En cuanto a la región dorso glútea las investigaciones describieron anatómica existente entre el músculo glúteo máximo y el nervio ciático. Esa conformación merece referencias de investigaciones que abarquen reflexiones sobre la red vascular local. 
La región del lado lateral del muslo surge en la región como un modelo de delimitación tradicional, y con mayor prioridad para la porción distal del rectángulo, debido a una menor distribución de los ramos de los nervios cutáneos laterales del muslo, que cuando transfijados por la punción intramuscular desencadenan un estímulo álgico intenso.

Por lo tanto, los conocimientos de las estructuras anatómicas se presentan indisociables de la práctica de administración de medicinas por vía IM, cabiendo al profesional enfermero a partir de un razonamiento clínico-reflexivo, adecuar el desarrollo de la técnica a la realidad presentada por el cliente.

\section{REFERENCIAS}

1 - Dangelo JG, Fattini CA. Anatomia Humana Sistêmica e Segmentar. 3a ed. São Paulo: Atheneu, 2007.

2 - Giovani AMM, organizadora. Cálculo e administração de medicamentos. $12^{\underline{a}}$ ed. São Paulo: Scrinium, 2006.

3 - Pereira AL, Bachion MM. Atualidades em revisão sistemática de literatura, critérios de força e grau de recomendação de evidência. Rev Gaúcha Enferm [periódico na internet]. 2006 dez [acesso em 2012 jan 14];27(4):491-8. Disponível em: http://seer.ufrgs.br/RevistaGauchadeEnfermagem/article/view/4633/2548

4 - Galvão CM, Sawada NO, Trevizan MA. Revisão Sistemática: recurso que proporciona a incorporação das evidências na prática da enfermagem. Rev Latino-am Enfermagem [periódico na internet]. 2004 mai-jun [acesso em 2012 jan 14];12(3):54956. Disponível em: http://www.scielo.br/pdf/rlae/v12n3/v12n3a14.pdf

5 - Berwanger O, Suzumura EA, Buehler AM, Oliveira JB. Como avaliar Criticamente Revisões sistemáticas e Metanálises? Revista Brasileira de Terapia Intensiva [periódico na internet]. 2007 out-nov [acesso em 2012 jan 14];19(4):475-480. Disponível em: http://www.scielo.br/pdf/rbti/v19n4/a12v19n4.pdf

6 - Godoy S, Nogueira MS, Mendes IAC. Aplicação de medicamentos por via intramuscular: análise do conhecimento entre profissionais de enfermagem. Rev Esc Enferm USP [periódico na internet]. 2004 [acesso em 2012 jan 14]; 38(2):135-42. Disponível em: http://www.scielo.br/pdf/reeusp/v38n2/03.pdf

7 - Meireles H, Motta-Filho GR. Lesão do nervo axilar causada pela injeção intramuscular no deltóide: relato de caso. Revista Brasileira Ortopedia. 2004;39(10):615-619.

8 - Moore KL, Dalley AF. Anatomia orientada para clínica. 4aa ed. Rio de Janeiro: Guanabara Koogan SA, 2001.

9 - Duque FL, Chagas CA. Acidente por injeção medicamentosa no músculo deltoide: lesões locais e à distância, revisão de 32 casos. J Vasc Bras [periódico na internet]. 2009 mar-jul [acesso em 2012 jan 14];8(3):238-246. Disponível em: http://www.scielo.br/pdf/jvb/v8n3/v8n3a09.pdf

10 - Godoy S. Educação em serviço por meio de vídeo conferência: aplicação de injetáveis via intramuscular na região ventro-glútea. [dissertação]. Ribeirão Preto (SP): Escola de Enfermagem de Ribeirão Preto, Universidade de São Paulo; 2002.

11 - Meneses AS, Marques IR. Proposta de um modelo de delimitação geométrica para injeção ventro-glútea. Revista Brasileira de Enfermagem [periódico na internet]. 2007 set-out [acesso em 2012 jan 14];60(5):552-8. Disponível em: http://www.scielo.br/pdf/reben/v60n5/v60n5a13.pdf

12 - Mainardes KJ. Administração de medicamentos por via intramuscular revisando uma prática farmacêutica. [monografia]. Maringá: Curso de Pós-Graduação em Farmacologia - Centro Universitário de Maringá; 2003. 
13 - Figueiredo NMA, organizadora Administração de medicamentos: revisando uma prática. Difusão Paulista de enfermagem, 2003.

14 - Mussi NM, Ohnishi M, Utyama IKA, Oliveira MMB. Técnicas fundamentais de enfermagem. São Paulo: Atheneu, 2003.

15 - Rocha RP, Fernandes GJM, Vengjer A, Mongon MLD, Ribeiro FP, Silva RBL. Distribuição do nervo cutâneo lateral da coxa na área de injeção intramuscular. Rev Assoc Med Bras [periódico na internet]. 2002 [acesso em 2012 jan 14];48(4):353-6. Disponível em: http://www.scielo.br/pdf/ramb/v48n4/14208.pdf, 